\title{
Technology-Supported Group Activity to Promote Communication in Dementia: A Protocol for a Within-Participants Study ${ }^{\dagger}$
}

\author{
Sarah K. Smith ${ }^{1, *(1)}$ and Arlene J. Astell 2,3,4 \\ 1 Interdisciplinary Centre of the Social Sciences, University of Sheffield, Sheffield S1 4DP, UK \\ 2 Ontario Shores Centre for Mental Health Sciences, Whitby, ON L1N 5S9, Canada; a.astell@reading.ac.uk \\ 3 Occupational Therapy \& occupational Sciences, University of Toronto, Toronto, ON M5S 1A1, Canada \\ 4 Berkshire Memory \& Cognition Research Centre, University of Reading, Reading RG6 6AH, UK \\ * Correspondence: s.k.smith@sheffield.ac.uk; Tel.: +44-(0)114-215-9120 \\ + This paper is an extended version of our paper published in AAATE2017 Congress Proceedings, Sheffield, \\ UK, 13-14 September 2017.
}

Received: 24 January 2018; Accepted: 6 March 2018; Published: 12 March 2018

\begin{abstract}
Computer Interactive Reminiscence and Conversation Aid (CIRCA)is an interactive conversation support for people living with dementia. CIRCA facilitates one-to-one conversations and caregiving relationships in formal care environments. Originally developed as a standalone device, a new web-based version of CIRCA has been created to increase availability. The potential of CIRCA to support group activities and conversation between people living with dementia and a facilitator has not previously been explored. The two objectives of this study are (i) to validate the new web-based version of CIRCA against the original standalone device, and (ii) to explore the efficacy of CIRCA in supporting group activity for people with dementia in a formal care setting. This mixed-methods study comprises two parts: (i) an eight-session group activity using the CIRCA stand-alone device, and (ii) an eight-session group activity using the web-based CIRCA. One hundred and eighty people with dementia will be recruited: 90 for part (i) and 90 for part (ii). Measures of cognition and quality of life will be taken at the baseline, post-CIRCA intervention, and three months later, plus video recordings of the group sessions. Both parts of the study will be completed by June 2018. The study will provide evidence on two issues: (i) a validation of the new web-based version of CIRCA, and (ii) the suitability of CIRCA to support group activities in formal care settings for people living with dementia. This protocol is an extended version of the short paper presented at the AAATE 2017 conference and published in Studies in Health Technology \& Informatics.
\end{abstract}

Keywords: dementia; psychosocial; Computer Interactive Reminiscence and Conversation Aid (CIRCA); technology

\section{Introduction}

Dementia is an overarching term, applied to a collection of progressive neurological disorders that at present cannot be prevented or reversed. Age is the biggest risk factor for developing dementia [1], and as life expectancy continues to grow, the number of people in the world with dementia is predicted to rise from 46.8 million in 2015, to 74.7 million by 2030, and to 131.5 million by 2050 [1]. There is no single cause of dementia, and no single pattern to the way it affects people. Alzheimer's disease (AD) is the most common cause of dementia, accounting for an estimated $60-80 \%$ of cases, with another $10 \%$ attributed to vascular causes (e.g., stroke-many of those with Alzheimer's pathology also have evidence of vascular disease [2]. Although there is a class of drugs—cholinesterase inhibitors-that can 
slow the progression of $\mathrm{AD}$, there are no disease-modifying therapies, i.e., nothing to reverse or cure any type of dementia [3], meaning that people will live with the condition for the rest of their lives.

Dementia is primarily a cognitive disorder [4] with each type of dementia characterised by a different profile of spared and impaired cognitive function. These can include memory, language, attention, planning, and initiating activities. For example, in AD, it has long been noted that an early symptom is a problem with working memory, which makes it difficult for people to keep current or recent events in mind [5]. However, their memory for people, places, and events from earlier in their lives, particularly childhood and early adulthood, is typically preserved [6]. Even so, dementia is a progressive disorder, and over time, all aspects of cognitive function are generally implicated. These changes affect people's social and emotional behaviour and everyday activities, making people with dementia increasingly reliant on family or professional caregivers for meeting all their needs [7].

The cognitive changes make communication between people with dementia and those who care for them particularly problematic. This is partly due to the working memory, which makes it difficult for people to hold conversations, as they cannot keep track of the turns [8]. In addition, repetitions and lack of responses can give the appearance of not listening to what their conversation partner is saying. This can be frustrating and disempowering for people with dementia, and distressing for the family and care staff they are trying to communicate with [9]. Developing interventions to support communication and maintain relationships between people with dementia and those who care for them is unsurprisingly a growing social and healthcare priority. In 2013, the U.K. government prioritised the identification of "non-drug interventions that make a real difference to people's lives and improve quality of life" and "enable people to maintain their dignity and independence for longer, reducing disability" [10] (p. 35). Whilst many non-pharmacological interventions for dementia have been developed and tested, to date only one-Cognitive Stimulation Therapy (CST) $[11,12]$ has been recommended by the U.K. National Institute of Health and Care Excellence (NICE). To stimulate the development, testing and implementation of new interventions, the Prime Minister's Challenge on Dementia 2020 highlighted the gaps in provision and the importance of offering an expanded range of services from the point of diagnosis. This pointed to approaches beyond pharmacology, specifically "incorporating new ideas including technology solutions into everyday practice" [13] (p. 32).

\subsection{Information and Communication Technologies (ICTs)}

Technology is becoming common practice in dementia care, with interventions such as GPS tracking systems and home health monitoring proving popular with caregivers [14]. Many of these technologies address issues of safety and security or provide reassurance to caregivers, but may not address the needs and priorities of people with dementia [15]. These sorts of technologies have been developed in line with the traditional medical model of dementia, which focuses on neurodegeneration and loss of abilities [16-18]. This view has been criticised for being both deterministic and pessimistic [19], and is responsible for widely-held misconceptions that little or nothing can be done directly for people living with dementia $[20,21]$.

One consequence has been an attitude towards dementia that is excessively disabling of individuals with the condition [22]. Excess disability refers to the situation where an individual with an acquired or congenital condition is disabled to a greater extent than is due to simply having the condition [23]. This excessive disabling is down to environmental factors, including the attitudes and behaviour of those around the person, which results in overtaking tasks or not allowing the individual to do a range of things for themselves. These decisions, while usually well-meaning, are paternalistic and not helpful for enabling individuals with dementia to retain their skills, autonomy, and independence [24]. In addition, this disabling attitude has meant that progress in developing technologies for people with dementia has been both slow and sparse, compared with the rapid developments in consumer electronics over the past 25 years.

In contrast to the medical model, the psychosocial approach focuses on the skills and abilities—both cognitive and behavioural—-that people with dementia retain. In addition, this approach 
takes account of the desires of people with dementia to continue participating in activities that are important to them [25]. These may very well be everyday activities, such as shopping and cooking, but also include socialising, hobbies, and leisure pursuits [24]. The potential of contemporary information and communication technologies (ICTs) to meet these needs and aspirations is increasingly being recognised in the dementia literature [26-28] with an exciting influx of evidence demonstrating how new forms of ICT can engage a person with dementia in activities they value [29-31]. This focus on the person with dementia as a separate entity from caregivers, who have their own different needs and priorities, is essential for examining the potential of contemporary ICTs to benefit them in line with the rest of the population [10,32].

Everyday non-specialised technology, such as television sets and telephones, have been shown to have utility and significance in the lives of people with dementia [27]. Further exploration of the growing mainstream functionality, including smart devices such as tablets, could go a long way to addressing the pressing needs of growing numbers of people with dementia. For example, individuals with dementia can be trained and supported to use tablets and smart devices to engage with activities of their choice and interest, just like the population at large [32].

The potential of technology to support people living with dementia is increasingly acknowledged, but currently far from realised [33]. One explanation is that opportunities, places, and support that may enable participation in such activities are currently not keeping pace with the desire from people living with dementia [32]. A further explanation is the slow pace of development of devices aimed at people with dementia rather than caregivers [24]. Coupled with these challenges, many people with dementia, along with their families and the services that support them, are largely unaware of the way ICT can be used, or unsure how to adopt it. There is also a need to expand research across the range of environments and contexts where people with dementia live and operate, to identify their needs and priorities for technological solutions both inside and outside the home.

\subsection{Computer Interactive Reminiscence and Conversation Aid (CIRCA)}

In addition to off-the-shelf devices and applications, there is room for bespoke interventions developed specifically to address the needs of people living with dementia. Computer Interactive Reminiscence and Conversation Aid (CIRCA) is a multimedia computer system developed to support and promote communication between people with dementia and caregivers [34,35]. CIRCA is based on reminiscing: the process of recollecting memories from one's life, about work or hobbies for instance, and speaking about these with one or more other people. Reminiscing is a basic human activity that emerges early in life and plays an important role in shaping self-identity and social relationships [36]. Telling our stories also provides a way to process and deal with emotional experiences, by allowing us to reflect on what has happened to us: "Our past experiences are emotionally saturated: we recount and reminisce about particular events because they are personally significant and emotionally meaningful" [37] (p. 39). For people living with dementia, engaging in reminiscing has shown to contribute to well-being and provide a positive activity [38].

Reminiscing is a popular group activity in dementia care settings, where one or more care staff or activity coordinators act as facilitators. Such sessions require facilitators to generate topics and find and organise materials to prompt reminiscing and discussion within the group [39]. It should be acknowledged that reminiscing is rarely carried out as a one-to-one activity in care settings, due to time constraints and the need to find stimuli for individuals.

CIRCA is a cooperatively-designed interactive software application comprising a multimedia database, containing a broad range of stimuli to prompt reminiscing by people with dementia [40]. It is delivered on a touch screen to avoid the need for a mouse, keyboard, prior experience, or intensive training. CIRCA utilises hypermedia to allow users to move instantaneously between different media including music, video clips, and photographs. CIRCA was designed to mitigate the working memory problem common in dementia by focusing on whatever is currently on screen, and removing the need to keep track of conversational turns [41]. Two key features of hypermedia are pertinent: first, 
the inherent flexibility enables users to explore interconnected but individual items as they choose, and there is no right or wrong way to navigate the materials. Second, hypermedia provides a dynamic opportunity involving photographs, music, and video to be seamlessly intertwined, resulting in an engaging shared experience [1,2]. Figure 1 illustrates a photograph being viewed on CIRCA.

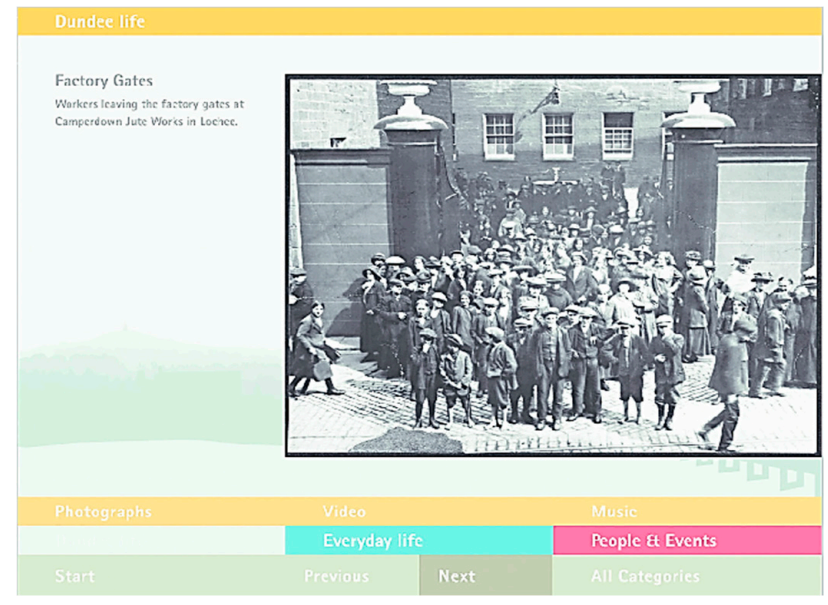

Figure 1. An example of a typical photograph included in Computer Interactive Reminiscence and Conversation Aid (CIRCA).

CIRCA was developed through a user-centred design approach, in order to address the expressed need for supported conversations between people with dementia and caregivers. Working closely with individuals living with dementia, family members in a caregiving role, and formal caregivers in a range of dementia care services, CIRCA was iteratively developed over a number of years [42]. All aspects of decision making relating to the interface, layout colours, fonts, backgrounds, buttons, and contents were informed through an interactive process consistently involving the intended user's perspectives and opinions [42].

The CIRCA team demonstrated that when compared to traditional reminiscing activities, CIRCA provided a more enjoyable experience for people with dementia and caregivers [15]. For the person with dementia, CIRCA provides the opportunity to make independent choices and engage as an equal partner during conversations with others [41]. Furthermore, the randomised presentation keeps caregivers engaged, and seeing people with dementia interacting with the computer leads them to re-evaluate their expectations of the person with dementia and view them "in a new light" [41]. Formal caregivers reported feelings of increased competence within the caring role, and were visibly more relaxed, resulting in time spent together using CIRCA improving their caregiving relationships [7].

\subsection{Current Study}

CIRCA was primarily developed to support one-to-one conversations between people living with dementia and caregivers. However, in most care settings there is little opportunity for one-to-one time, and so group activities are favoured. In a pilot study across two care homes, caregivers utilised CIRCA in a wide range of activities besides one-to-one interactions, including groups, quizzes, and getting to know new residents [42]. In spite of this creativity, there has been no systematic application or exploration of CIRCA as a group activity that could be scalable across care settings. The current study was developed firstly to examine the utility of CIRCA as a systematic group activity that can be delivered in a range of care settings. The second purpose was to validate a new web-based version of CIRCA (CIRCA-WB) against an existing version, prepopulated on a standalone device. The existing CIRCA version is populated with generic material that may be suitable for the majority. Figure 2 illustrates CIRCA being explored and discussed. 


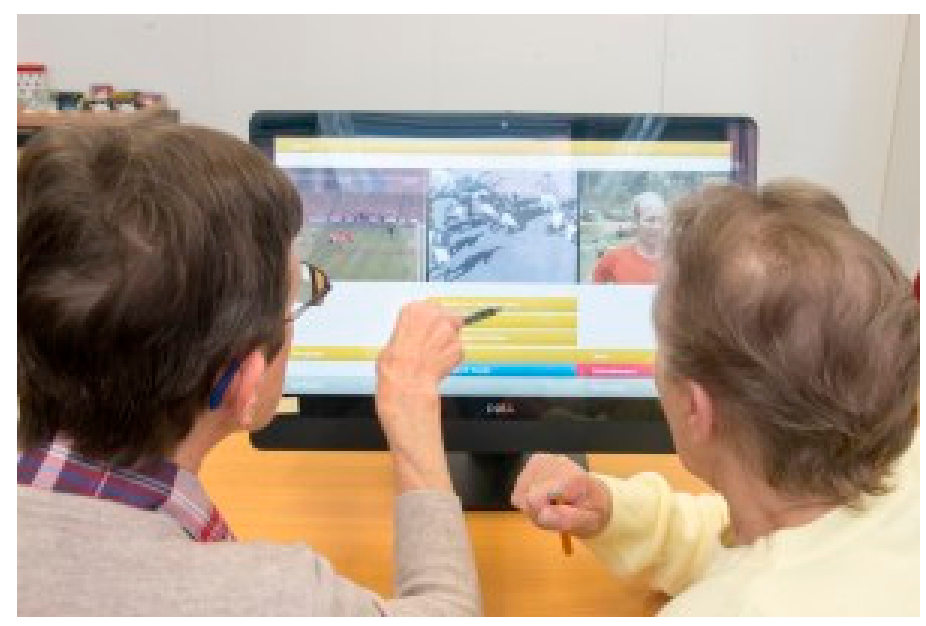

Figure 2. The original CIRCA being enjoyed.

CIRCA-WB is being developed as part of the Independent Living Functions for the Elderly (IN-LIFE) project, a large European Horizon 2020 program of work developing a cloud-based platform for people with dementia and their caregivers across Europe to access. CIRCA-WB retains the look and feel of CIRCA, with similar backgrounds and layouts to the original. The randomised presentation has been retained to provide as close an experience to the original CIRCA as possible. The web-based contents are stored in the cloud and accessed via a login. CIRCA-WB has the added functionality that contents can be added or labeled in a wide range of languages, ensuring that it can be used across countries and topics. For the U.K. pilot site, CIRCA-WB will be localised to Sheffield, the city in which the majority of participants live. This will involve accessing the Sheffield Archives for local material about the city and the surrounding areas.

CIRCA-WB will be offered as part of the IN-LIFE platform, along with 18 other web-based services to support people living well with dementia. These alternate services are being investigated in parallel across five other European countries, to explore the potential of digital services for supporting people to live well with dementia using contemporary ICTs.

The protocol development was informed by examining the work of Spector et al [11,12] in developing and testing Cognitive Stimulation Therapy (CST) as a group-based activity. CST is a manualised activity program developed from the principles of reality orientation and "cognitive stimulation" [43] to enhance cognitive function and quality of life for people living with dementia. CST was developed as an intervention for use in day programs and care homes and tested in a single-blind, multi-centre, randomised controlled trial [11]. The findings demonstrated an improvement in both the cognitive performance and quality of life of participants who received CST, relative to those who did not, and the improvements "compare favourably with trials of drugs for dementia" [13] (p. 248). A cost-effectiveness review of a 16-session CST program twice a week for eight weeks suggested it could be more cost-effective than usual treatment [44]. This current protocol will enable the systematic study of CIRCA as a group intervention, whilst comparing the existing application with that of a web-based version developed for the IN-LIFE project. We also intend to compare the findings with those achieved by CST, as CIRCA requires minimal training or set up and could provide a technology-based alternative to CST in dementia care settings.

\section{Methods}

\subsection{Design}

This protocol describes a within-participant's design, where all participants will receive the intervention. All participants will be assessed at baseline, at the end of the intervention, and three 
months later, to explore the possible maintenance of effects of the intervention. The project comprises two parts: (i) an eight-session standalone CIRCA-based group activity, and (ii) an eight-session web-based CIRCA-based group activity.

\subsection{Sample Size}

The primary outcome will be the impact of CIRCA on cognitive function. The Addenbrookes Cognitive Examination - third edition (ACE-III) will be used to assess cognition at three time points: baseline, at the end of the intervention, and three months post-intervention. To detect a standardised mean change or effect size of 0.25 on the ACE-III outcome, with $90 \%$ power and $5 \%$ (two-sided) significance level, and assuming $15 \%$ attrition, a minimum sample size of 180 people with dementia will need to be recruited.

\subsection{Ethics}

Health Research Authority (HRA) approval was received and a favourable opinion from the Sheffield Research Ethics Committee(REC), reference 16/YH/0354.

\subsection{Participants}

A total of 180 older adults living with cognitive impairment will be recruited from Sheffcare Ltd. (a provider of residential care and day care), and Alzheimer's Society Sheffield. Half of the participants will take part in an eight-session (twiceweekly for four weeks) group activity using standalone CIRCA, and half will take part in an eight-session (twiceweekly for four weeks) group activity using web-based CIRCA (CIRCA-WB; see Figure 3).

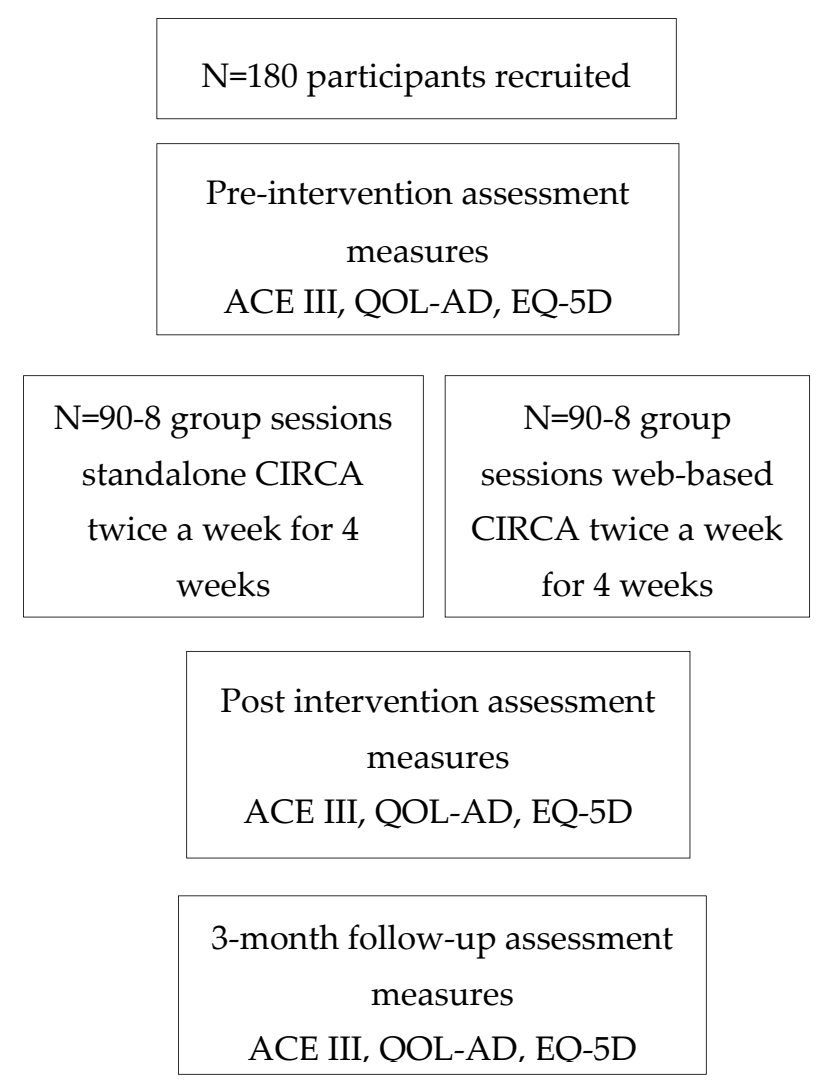

Figure 3. Recruitment of participants and intervention applied. Addenbrookes Cognitive Examination-III (ACE-III); The quality of life in Alzheimer's disease (QOL-AD); EuroQol-5D (EQ-5D). 


\subsection{Recruitment Strategy}

Recruitment will be supported by the Sheffield Alzheimer's Society dementia support workers and day care managers at Sheffcare, who will assist in the identification of potential participants with their knowledge and expertise of their client's respective organisations. Each organisation will be provided with information sheets and study flyers to distribute to potential participants who meet the inclusion criteria (see Table 1). A follow up telephone call to each organisation will follow 2-3 days later, to identify people interested in participating. At this time, the details of those interested individuals will be passed to the researcher for further contact.

Table 1. Inclusion/exclusion criteria people living with dementia.

\begin{tabular}{cc}
\hline Inclusion Criteria & Exclusion Criteria \\
\hline $\begin{array}{c}\text { Diagnosis of dementia according to the Diagnostic \& } \\
\text { Statistical Manual of Mental Disorders(DSM-5) }\end{array}$ & Requires consultee involvement \\
\hline Ability to verbally communicate & $\begin{array}{c}\text { Sensory impairment that will negatively impact } \\
\text { technology participation }\end{array}$ \\
\hline & $\begin{array}{c}\text { Secondary long term conditions that could affect } \\
\text { group participation }\end{array}$ \\
\hline & $\begin{array}{c}\text { Behavioural challenges that could negatively affect } \\
\text { group activities }\end{array}$ \\
\hline
\end{tabular}

\section{Materials}

\subsection{Assessment Measures}

To minimise burden on participants with dementia, only three measures will be used.

\subsection{Cognition}

The primary outcome measure is the Addenbrookes Cognitive Examination-III (ACE-III) [45]. The ACE-III covers five cognitive domains; attention, memory, fluency, language, and visuospatial —and is scored out of 100. The ACE-III cognitive domains have been found to correlate significantly with standardised neuropsychological tests illustrating high levels of sensitivity and validity. For comparison with the CST study [12], the ACE-III scores will be converted to equivalent Mini Mental State Examination(MMSE) scores, using the algorithm developed by Giebeland Challis (2017) [46].

\subsection{Quality of Life}

The quality of life in Alzheimer's disease (QOL-AD) [47] scale is the secondary outcome measure. The QOL-AD comprises thirteen items, covering physical health, energy, mood, living situation, memory, family, marriage, friends, chores, fun, money, self, and life as a whole. QOL-AD can be completed by people with a range of dementia severity. QOL-AD was also used by Spector et al. (2003) [11] in their CST study, where they found it has good internal consistency, validity, and reliability [11]. QOL-AD is recommended by the European consensus on outcome measures for psychosocial interventions in dementia [48].

\subsection{Health Status}

The EQ-5D [49], is an additional, brief, self-reported health and quality of life measure, which is included as a further secondary measure. The EQ-5D has been widely used with a range of study populations, and has been considered appropriate to utilise with people with dementia $[50,51]$ producing a simple value of health status. 


\section{Procedure}

\subsection{CIRCA Group Activity}

One hundred and eighty participants with dementia will be recruited from Sheffcare Ltd., Sheffield, UK and the local Alzheimer's Society in Sheffield, UK. Within Sheffcare, half of the participants will be recruited from the day care program and half from residential care homes. The Alzheimer's Society participants will all be people living at home in the community. It is considered that this will give a varied representation of the stage and experience of dementia. The goal is for all participants to provide independent informed and maintained consent, with a full understanding of the nature and purpose of the research project. Obtaining informed consent from people with dementia is an essential part of the principle of autonomy [52] (p. 305), and will be taken in accordance with the Mental Capacity Act of 2005. In order to ensure that the researcher effectively gains and maintains consent from participants, the "process consent model" [53] will be closely adhered to. This will include accessible consent forms and ongoing consent monitoring, as well as feedback and support.

Following the consent procedure, each participant will complete the three formal measures (ACE-III, QoL-AD, and EQ-5D) in the week prior to the first group session. A caregiver can be present if they choose during the baseline assessment. Participants will be recruited to participate in small groups of between 4-6 individuals. Over the course of the project, between 30 and 45 groups will be required. The group intervention will run twice a week for $60 \mathrm{~min}$, over 4 weeks, and consist of the group and one facilitator interacting with CIRCA. Figure 4 illustrates an example image of what a group of 5 participants plus the facilitator may look like.

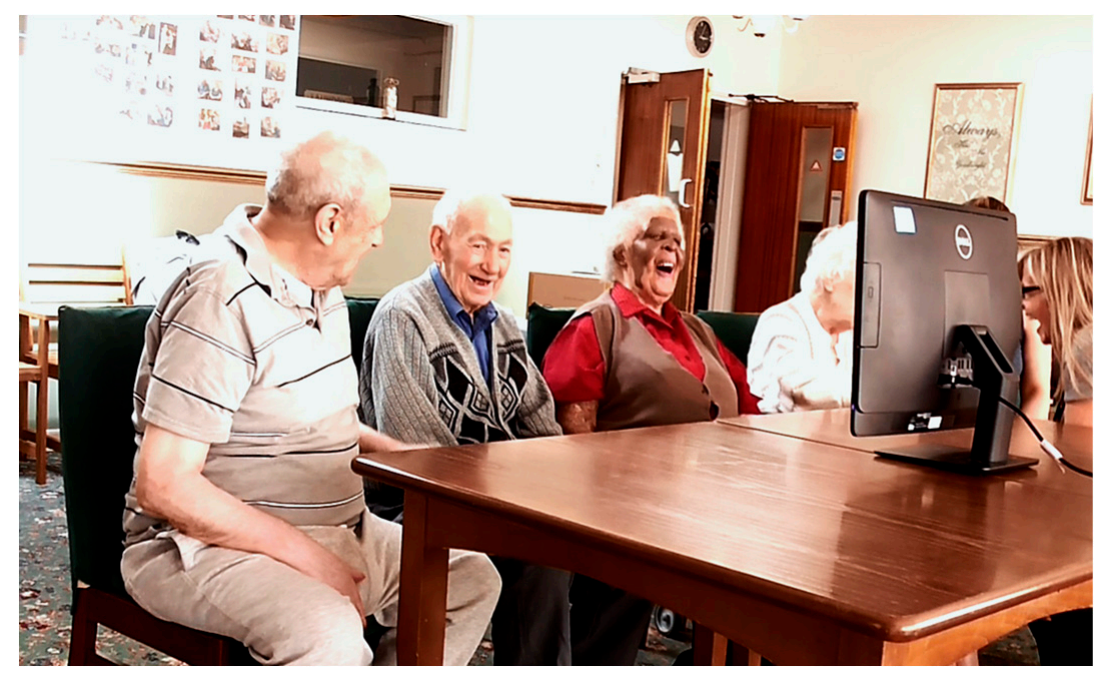

Figure 4. An example of group interactions involving CIRCA.

Half of the groups will use the original standalone CIRCA and half will use CIRCA-WB. All of the group sessions will be video-recorded to examine the group dynamics and social interactions. All group members will be seated facing the $21.5^{\prime \prime}$ desktop touch-screen computer displaying CIRCA, and the facilitator will be seated to one side of the screen. All sessions will take place in quieter areas away from ongoing activities, to reduce noise and distraction for the group. At the beginning of each session, the facilitator will seek ongoing verbal consent and ensure each member of the group understands why they are there and what they are going to do. The session will begin with the facilitator offering the group the choice of "music, video, or photographs", followed by the topic, which could be any of six choices: entertainment, childhood, sport, everyday life, recreation, people, and events. Following the eighth and final session, the formal measures (ACE-III, QoL-AD, and EQ-5D) will be repeated. Three months after the end of the group sessions, the three measures (ACE-III, QoL-AD, EQ-5D) will 
be repeated. Two researchers will be involved in completing the formal assessments and conducting the sessions. Where Researcher (1) undertakes the assessments at baseline, post-CIRCA, and three months later, Researcher (2) will facilitate the eight group sessions, and vice versa.

\subsection{Analysis}

Repeated measures analysis of variance (ANOVA) will be used to examine the impact of CIRCA on the primary outcome measure (ACE III) by comparing scores at baseline, post-intervention, and three months later. Post-hoc analysis will be carried out to explore any significant relationships.

Repeated measures ANOVA will also be used to examine the impact of CIRCA on the two secondary outcome measures (QoL-AD and EQ-5D). Post-hoc analysis will also be conducted as appropriate.

To validate CIRCA-WB against the original CIRCA, a Bland-Altman analysis [54] will be used to compare the cognitive (ACE-III) outcomes between the two versions.

\section{Discussion}

This protocol has been designed to address the identified need for new provisions for people living with dementia utilising contemporary ICTs. CIRCA has been extensively evaluated within smaller projects since its development, but not as a group activity at scale. The intention is to further develop CIRCA and ultimately present it as a web-based application accessible through the IN-LIFE cloud-based platform, as part of a large European multi-site trial. As part of IN-LIFE, our partners in the Netherlands, Spain, and Sweden are also piloting culturally and linguistically-appropriate versions of CIRCA. If successful, the trial results will contribute to existing research and practice regarding the "best" ways to engage people with dementia using contemporary ICTs. Furthermore, positive outcomes could also provide additional options for services to offer, once a diagnosis has been received.

\section{Trial Status}

The trial is ongoing and will be completed in June 2018.

Acknowledgments: Independent Living Functions for the Elderly (IN-LIFE) is a Horizon 2020 funded project; Grant Agreement No. 643442.

Author Contributions: Sarah K. Smith and Arlene J. Astell conceived and designed the experiments; Sarah K. Smith performed the experiments; Sarah K. Smith and Arlene J. Astell analyzed the data and wrote the paper.

Conflicts of Interest: The authors declare no conflict of interest.

\section{References}

1. Prince, M.; Albanese, M.; Guerchet, E.; Prina, M. World Alzheimer Report; Alzheimer's Disease International: London, UK, 2014.

2. Alzheimer's Association. 2017 Alzheimer's Disease Facts and Figures; Alzheimer's Association: Chicago, IL, USA, 2017.

3. Egan, K.; Macleod, M. Two decades testing interventions in transgenic mouse models of Alzheimer's disease: Designing and interpreting studies for clinical trial success. Clin. Investig. 2014, 4, 693-704. [CrossRef]

4. American Psychiatric Association. Diagnostic and Statistical Manual of Mental Disorders (DSM-5), 5th ed.; American Psychiatric Association Publishing: Washington, DC, USA, 2013.

5. Baddeley, A.; Logie, R.; Bressi, S.; della Sala, S.; Spinnler, H. Dementia and Working Memory. Q. J. Exp. Psychol. Sect. A 1986, 38, 603-618. [CrossRef]

6. Gold, C.A. Memory loss in Alzheimer's disease: Implications for development of therapeutics. Expert Rev. Neurother. 2009, 8, 1879-1891. [CrossRef] [PubMed] 
7. Astell, A.J.; Ellis, M.P.; Bernardi, L.; Alm, N.; Dye, R.; Gowans, G.; Campbell, J. Using a touch screen computer to support relationships between people with dementia and caregivers. Interact. Comput. 2010, 22, 267-275. [CrossRef]

8. Alm, N.; Dye, R.; Gowans, G.; Campbell, J.; Astell, A.; Ellis, M. A communication support system for older people with dementia. Computer 2007, 40, 35-41. [CrossRef]

9. Searson, R.; Hendry, A.M.; Ramachandran, R.; Burns, A.; Purandare, N. Activities enjoyed by patients with dementia together with their spouses and psychological morbidity in carers. Aging Ment. Health 2008, 12, 276-282. [CrossRef] [PubMed]

10. Department of Health. Dementia: A State of the Nation Report on Dementia Care and Support in England; Department of Health: London, UK, 2013.

11. Spector, A.; Thorgrimsen, L.; Woods, B.; Royan, L.; Davies, S.; Butterworth, M.; Orrell, M. Efficacy of an evidence-based cognitive stimulation therapy programme for people with dementia: Randomised controlled trial. Br. J. Psychiatry 2003, 183, 248-254. [CrossRef] [PubMed]

12. Spector, A.; Orrell, M.; Davies, S.; Woods, B. Can reality orientation be rehabilitated? Development and piloting of an evidence-based programme of cognition-based therapies for people with dementia. Neuropsychol. Rehabil. 2001, 11, 377-397. [CrossRef]

13. Department of Health. The Dementia Challenge: Driving Improvements in Health; Department of Health: London, UK, 2015.

14. Burstein, A.A.; DaDalt, O.; Kramer, B.; D'Ambrosio, L.A.; Coughlin, J.F. Dementia caregivers and technology acceptance: Interest outstrips awareness. Gerontechnology 2015, 14, 45-56. [CrossRef]

15. Astell, A.J. Technology and personhood in dementia care. Qual. Ageing 2006, 7, 15-25. [CrossRef]

16. Gilliard, J. Dementia care in England and the social model of disability: Lessons and issues. Dementia 2005, 4, 571-586. [CrossRef]

17. Innes, A. Dementia Studies: A Social Science Perspective; SAGE Publications Ltd.: Thousand Oaks, CA, USA; London, UK, 2009.

18. The National Institute for Health and Care Excellence. Dementia: Supporting People with Dementia and Their Carers in Health and Social Care; The National Institute for Health and Care Excellence: London, UK, 2006.

19. Kitwood, T. Dementia Reconsidered: The Person Comes First; Open University Press: Buckingham, UK, 1997.

20. Banerjee, S. Living well with dementia-Development of the national dementia strategy for England. Int. J. Geriatr. Psychiatry 2010, 25, 917-922. [CrossRef] [PubMed]

21. Vernooij-dassen, M.J.; Moniz-Cook, E.D.; Woods, R.T.; De Lepeleire, J.; Leuschner, A.; Zanetti, O.; de Rotrou, J.; Kenny, G.; Franco, M.; Peters, V.; et al. Factors affecting timely recognition and diagnosis of dementia-From awareness to stigma 2005. Int. J. Geriatr. Psychiatry 2005, 20, 377-386. [CrossRef] [PubMed]

22. Wells, D.L.; Dawson, P. Description of retained abilities in older persons with dementia. Res. Nurs. Health 2000, 23, 158-166. [CrossRef]

23. Brody, H.; Kleban, E.; Lawton, M.; Silverman, P. Excess Disabilities of Mentally Impaired Aged: Impact of Individualized Treatment. Gerontologist 1971, 11, 124-133. [CrossRef] [PubMed]

24. Astell, A.J. Supporting a good life with dementia. In Ageing and the Digital Life Course; Prendergast, D., Garattini, C., Eds.; Berghan Books: Oxford, UK, 2015.

25. Smith, S.K.; Mountain, G.A. New forms of information and communication technology (ICT) and the potential to facilitate social and leisure activity for people living with dementia. Int. J. Comput. Healthc. 2012, 1, 332-345. [CrossRef]

26. Meiland, F.; Innes, A.; Mountain, G.; Robinson, L.; van der Roest, H.; García-Casal, J.A.; Gove, D.; Thyrian, J.R.; Evans, S.; Dröes, R.M.; et al. Technologies to Support Community-Dwelling Persons with Dementia: A Position Paper on Issues Regarding Development, Usability, Effectiveness and Cost-Effectiveness, Deployment, and Ethics. JMIR Rehabil. Assist. Technol. 2017, 4, e1. [CrossRef] [PubMed]

27. Nygard, L. The meaning of everyday technology as experienced by people with dementia who live alone. Dementia 2008, 7, 481-502. [CrossRef]

28. Topo, P. Technology Studies to Meet the Needs of People with Dementia and Their Caregivers: A Literature Review. J. Appl. Gerontol. 2008, 28, 5-37. [CrossRef]

29. Cutler, C.; Hicks, B.; Innes, A. Technology, fun and games. J. Dement. Care 2014, 22, 12-13. 
30. Leng, F.Y.; Yeo, D.; George, S.; Barr, C. Comparison of iPad applications with traditional activities using person-centred care approach: Impact on well-being for persons with dementia. Dementia 2014, 13, 265-273. [CrossRef] [PubMed]

31. Lazar, A.; Demiris, G.; Thompson, H.J. Involving family members in the implementation and evaluation of technologies for dementia: A dyad case study. J. Gerontol. Nurs. 2015, 41, 21-26. [CrossRef] [PubMed]

32. Smith, S.K. Exploring the Potential of Touch-Screen Computer Technology in Promoting Enjoyable Activities for People Living with Dementia: A Visual Ethnography Sarah Kate Smith a Thesis Submitted to the University of Sheffield in Partial Fulfilment of the Requiremen; University of Sheffield: Sheffield, UK, 2015.

33. Robinson, L.; Brittain, K.; Lindsay, S.; Jackson, D.; Olivier, P. Keeping in Touch Everyday (KITE) project: Developing assistive technologies with people with dementia and their carers to promote independence. Int. Psychogeriatr. 2009, 21, 494-502. [CrossRef] [PubMed]

34. Alm, N.; Astell, A.; Ellis, M.; Dye, R.; Gowans, G.; Campbell, J. A cognitive prosthesis and communication support for people with dementia. Neuropsychol. Rehabil. 2004, 14, 117-134. [CrossRef]

35. Astell, A. Facilitating communication in dementia with multimedia technology. Brain Lang. 2004, 91, 80-81. [CrossRef]

36. Astell, A.J.; Purves, B.; Phinney, A. "Story of My Life?" The Contents and Functions of Reminiscing. Available online: http:/ / www.cs.cornell.edu/ danco/remchiwork/papers/astell-Stimulating-reminiscingBP-AJA.pdf (accessed on 7 December 2017).

37. Fivush, E.; Haden, R.; Reese, C. Remembering, recounting and reminiscing: The development of autobiographical memory in social context. In Rembereing Our Past: Studies in Autobiographical Memory; Rubin, D.C., Ed.; Cambridge University Press: Cambridge, UK, 1995.

38. Fels, D.I.; Astell, A.J. Storytelling as a model of conversation for people with dementia and caregivers. Am. J. Alzheimers Dis. Other Dement. 2011, 26, 535-541. [CrossRef] [PubMed]

39. Schweitzer, E.; Bruce, P. Remembering Yesterday, Caring Today: Reminiscence in Dementia Care-A Guide to Good Practice UK; Jessica Kingsley: London, UK, 2008.

40. Gowans, G.; Dye, R.; Alm, N.; Vaughan, P.; Astell, A.; Ellis, M. Designing the interface between dementia patients, caregivers and computer-based intervention. Des. J. 2007, 10, 12-23. [CrossRef]

41. Astell, P.; Alm, A.J.; Gowans, N.; Ellis, G.; Dye, M.; Campbell, R.; Vaughan, J. The CIRCA: A communication prosthesis for dementia. Technol. Aging 2008, 105, 64-69.

42. Astell, M.S.; Parsons, A. CIRCA: Technology to prompt reminiscing and conversation between residents in care homes and care staff. Gerontechnology 2010, 9, 68-69. [CrossRef]

43. Breuil, V.; de Rotrou, J.; Forette, F.; Tortrat, D.; Ganansia-Ganem, A.; Frambourt, A.; Moulin, F.; Boller, F. Cognitive stimulation of patients with dementia: Preliminary results. Int. J. Geriatr. Psychiatry 1994, 9, 211-217. [CrossRef]

44. Knapp, M.; Thorgrimsen, L.; Patel, A.; Spector, A.; Hallam, A.; Woods, B.; Orrell, M. Cognitive stimulation therapy for people with dementia: Cost-effectiveness analysis. Br. J. Psychiatry 2006, 188, 574-580. [CrossRef] [PubMed]

45. Hsieh, S.; Schubert, S.; Hoon, C.; Mioshi, E.; Hodges, J.R. Validation of the Addenbrooke's Cognitive Examination III in Frontotemporal Dementia and Alzheimer's Disease. Dement. Geriatr. Cogn. Disord. 2013, 36, 3-4. [CrossRef] [PubMed]

46. Giebel, C.M.; Challis, D. Sensitivity of the Mini-Mental State Examination, Montreal Cognitive Assessment and the Addenbrooke's Cognitive Examination III to everyday activity impairments in dementia: An exploratory study. Int. J. Geriatr. Psychiatry 2017, 32, 1085-1093. [CrossRef] [PubMed]

47. Logsdon, R.G.; Gibbons, L.E.; McCurry, S.M.; Teri, L. Assessing Quality of Life in Older Adults with Cognitive Impairment. Psychosom. Med. 2002, 64, 510-519. [CrossRef] [PubMed]

48. Moniz-Cook, E.; Vernooij-Dassen, M.; Woods, R.; Verhey, F.; Chattat, R.; De Vugt, M.; Mountain, G.; O'Connell, M.; Harrison, J.; Vasse, E.; et al. A European consensus on outcome measures for psychosocial intervention research in dementia care. Aging Ment. Health 2008, 12, 14-29. [CrossRef] [PubMed]

49. Euroqol Group. EuroQol-A new facility for the measurement of health-related quality of life. Health Policy 1990, 16, 199-208.

50. Hounsome, N.; Orrell, M.; Edwards, R.T. EQ-5D as a quality of life measure in people with dementia and their carers: Evidence and key issues. Value Health 2011, 14, 390-399. [CrossRef] [PubMed] 
51. Wolfs, C.A.G.; Kessels, A.; Dirksen, C.D.; Severens, J.L.; Verhey, F.R.J. Integrated multidisciplinary diagnostic approach for dementia care: Randomised controlled trial. Br. J. Psychiatry 2008, 192, 300-305. [CrossRef] [PubMed]

52. Bjorneby, S. Ethical Considerations in the ENABLE Project. Dementia 2004, 3, 297-312. [CrossRef]

53. Dewing, J. Participatory research A method for process consent with persons who have dementia. Dementia 2007, 6, 11-25. [CrossRef]

54. Bland, J.M.; Altman, D.G. Statistical methods for assessing agreement between two methods of clinical measurement. Lancet 1986, 1, 307-310. [CrossRef]

(c) (C) 2018 by the authors. Licensee MDPI, Basel, Switzerland. This article is an open access article distributed under the terms and conditions of the Creative Commons Attribution (CC BY) license (http://creativecommons.org/licenses/by/4.0/). 